\title{
How to manage an intraoperative endoleak following left pneumonectomy with aortic wall en-bloc resection in an invasive lung cancer after aortic endoprosthesis insertion
}

\author{
Antonio Francisco Honguero Martínez ${ }^{1 *}$, Pablo León Atance ${ }^{1}$, Martín Landaluce Chaves ${ }^{2}$ and María Dolores García Jiménez \\ ${ }^{1}$ Thoracic Department, University General Hospital of Albacete, Spain \\ ${ }^{2}$ Thoracic and Vascular Departments, University General Hospital of Albacete, Spain
}

\begin{abstract}
Lung cancer can sometimes invade vital adjacent structures. When the descending thoracic aorta is involved, en-bloc resection can be achieved after previous deployment of aortic endoprosthesis. We describe an intraoperative type-I endoleak following left pneumonectomy for invasive lung cancer with en-bloc resection of a patch of the descending thoracic aorta. This serious complication was controlled by temporary attaching the buldging endoprosthesis to the aortic wall with a pledged stitch and insertion of another aortic endoprosthesis more proximal to the previous one.
\end{abstract}

\section{Introduction}

Lung cancer can sometimes invade vital adjacent structures and in selected cases surgical treatment with curative intent can be considered [1]. Resecting a lung tumor with invasion of the descending thoracic aorta (DTA) represents a difficult surgical procedure.

In the last years, partial aortic resection has been described in surgical treatment of lung cancer inserting previously an endovascular stent.

We report a case of en-bloc resection of lung cancer involving the DTA (Figure-1) and an intraoperative challenging complication due to a type-I endoleak, how it was managed and the posterior follow-up.

\section{Technique}

A left lung cancer with high suspicion of invasion of the DTA was diagnosed in a 59-year-old man. Surgical planification was to insert on supine position an aortic endoprosthesis with fluoroscopy guidance through the left femoral artery (Zenith TX2 38x152mm; Cook Inc., Bloomington, In. USA). The prosthesis was deployed $2.5 \mathrm{~cm}$ caudal from the origin of the left subclavian artery to have place to insert a vascular clamp in case of need during open surgical resection.

Afterwards and in the same anesthetic procedure, the patient was replaced on right lateral decubitus position. A standard posterolateral thoracotomy was the surgical approach and a left pneumonectomy with en bloc resection of a $2 \times 3 \mathrm{~cm}$-sized patch of the full thickness wall of the DTA was performed. The endoaortic prosthesis remained exposed through the newly created aortic wall defect (Figure 1). Then standard mediastinal lymphadenectomy was underwent. While tailoring a pericardial fat flap to cover the left bronchial stump a sudden aortic leak appeared through a 1-2 mm-sized defect on the proximal part of the endoprosthesis due to progressive herniation (type-1 endoleak). Digital compression impeded the bleeding while a pledged $3 / 0$ polypropilene stitch was used to fix the proximal edge of the endoprosthesis inside the aortic lumen. The stitch involved part of the proximal aortic wall and also the adjacent endoprosthesis. This way the bleeding was totally controlled. Nevertheless due to the risk of later progressive avulsion we decided to insert a second endoprosthesis to be deployed more proximal, just adjacent to the origin of the left subclavian artery (Zenith TX2 de 38x202mm (Cook Inc., Bloomington, Indiana, USA). The patient remained on lateral decubitus due to the delicated situation while a surgeon controlled and prevented a possible herniation of the endoprosthesis out of the aortic wall by gently grasping the compromised segment of the aorta.

After deployment of the second endoprosthesis vascular stability was achieved. Quantity of blood loss was $600 \mathrm{~mL}$ during the whole intervention. Two units of blood cells were transfused in the operating room. A mediastinal fat flap covered the bronchial stump. The patient was discharged 18 days later after recovering from accute confusional syndrome, and mild right lower limb monoparesia. No other postoperative complications appeared related to vascular and pulmonary procedures.

\section{Comments}

After performing the left pneumonectomy and resecting a patch of the DTA the endoprosthesis remained exposed through the aorta wall defect and no one expected it could be herniated, because aproximately $3 \mathrm{~cm}$ length of endoprosthesis still remained inside the DTA cranial to the proximal aortic wall defect. Only while performing a mediastinal fat flap to cover the left bronchial stump a sudden arterial blood leak was

Correspondence to: Antonio Francisco Honguero Martínez, Thoracic Department, University General Hospital of Albacete, C/. Hermanos Falcó 37. E-02006, Spain, Tel/Fax: 0034-967-597213; E-mail: tonyhonguero@yahoo.es

key words: aorta, vascular endoprosthesis, vascular endoleak, invasive lung cancer, surgery, pneumonectomy

Received: October 05, 2017; Accepted: October 24, 2017; Published: October 27,2017 
Martínez AFH (2017) How to manage an intraoperative endoleak following left pneumonectomy with aortic wall en-bloc resection in an invasive lung cancer after aortic endoprosthesis insertion

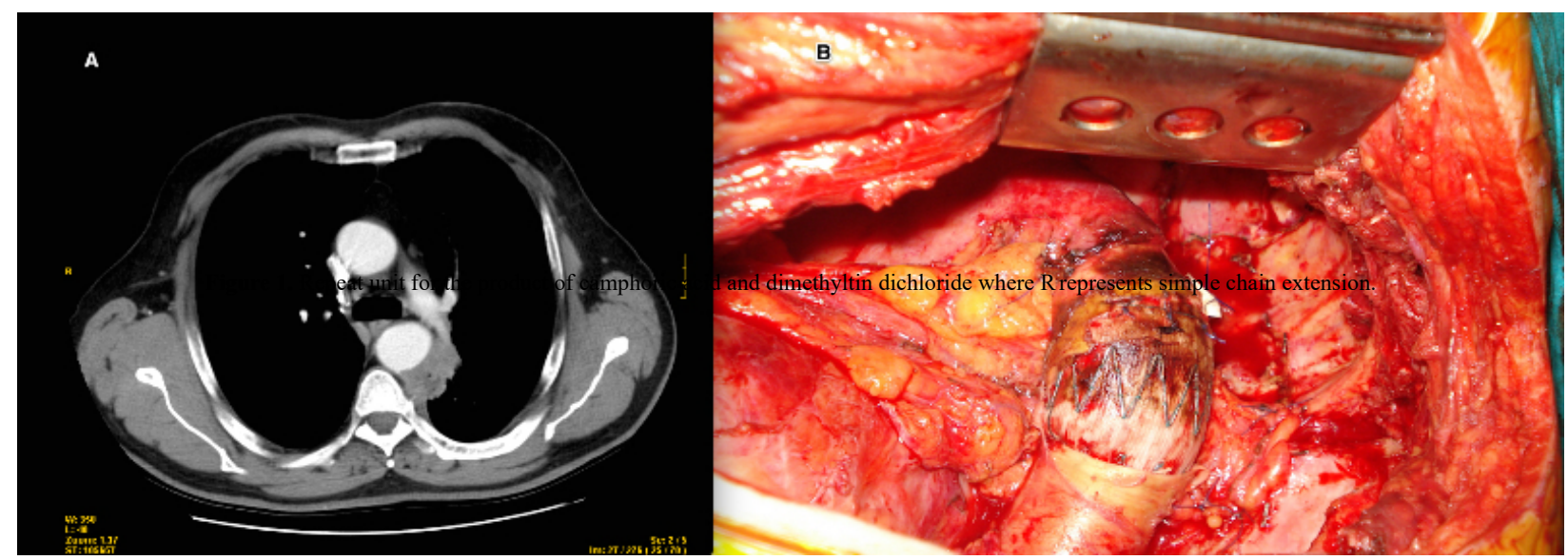

Figure 1. (A) CT-scan showing left pulmonary tumor invading the descending thoracic aorta. (B) Intraoperative photograph shows the descending thoracic aorta defect, exposing the endoprosthesis without any leak after pledged 3/0 polypropylene stitch and insertion of the second endoprosthesis.

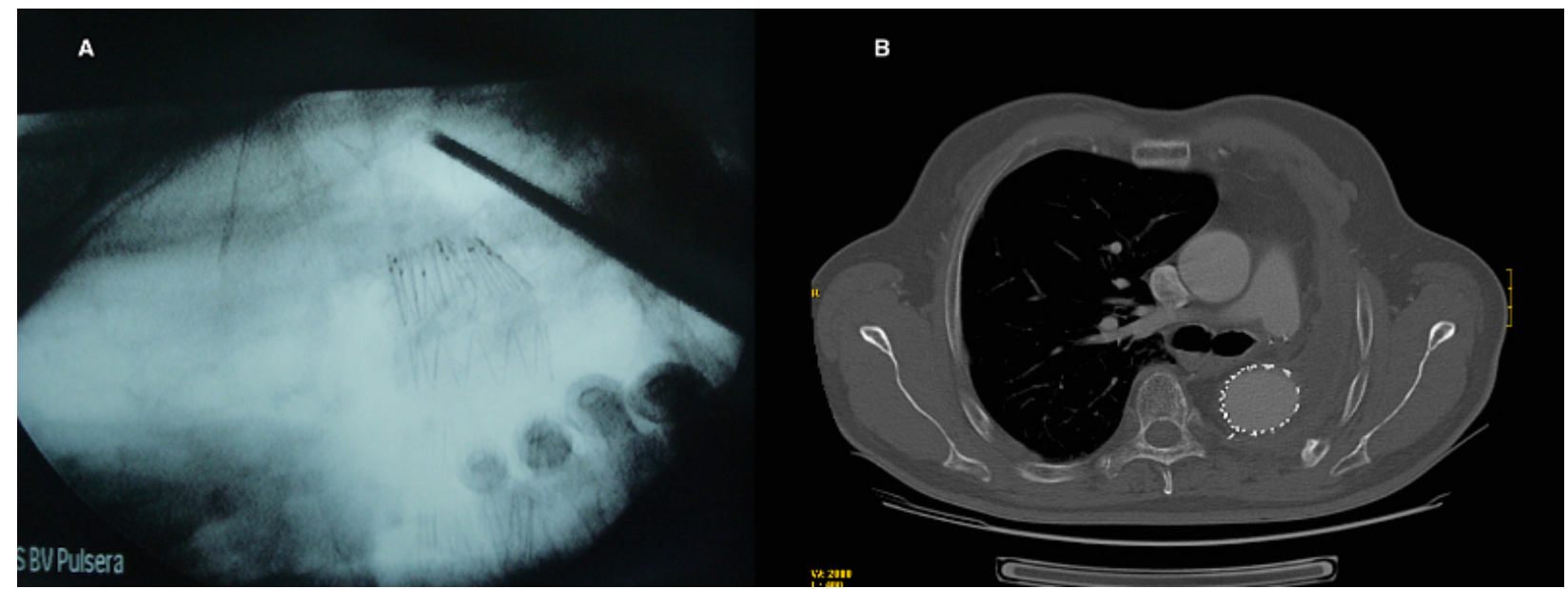

Figure 2. (A) Radioscopic image of a surgeon holding in place the herniated aortic endoprosthesis while a second one was been deployed. (B) Postoperative thorax CT-scan shows the two endoprosthesis placed in the thoracic descending aorta.

discovered. This was originated by progressive avulsion or herniation of the endovascular stent from its superior edge off the aortic defect.

The exact deployment of the stent was calculated on purpose to leave enough space just in case a vascular clamp on the aortic arch had to be placed during the open procedure. Inmediate application of a pledged 3/0 polypropylene stitch involving native aortic wall and the endoprosthesis was fundamental to impede progressive herniation, what would have dramatic consequences. Nevertheless grasping and protecting the aortic wall defect with the surgeon's hand was also important. Although insertion of the second endovascular stent was performed on lateral decubitus, femoral artery access was feasible due to the recent dissection and closure of inguinal wound. It is important to count on another endovascular stent adjusted to patient's measures just in case of urgent need as was our case. It is described that occlusion of left subclavian artery is sometimes a common procedure in aortic pathology treated by endovascular stent (aneurisms, accute aortic dissection) without major cerebrovascular neither left superior limb impairment [2]. It has been described ressection of median aortic wall layer leaving in place the aortic adventitia, although it is a very weak structure that would not have impeded endoprosthesis avulsion in our case [3]. Probably if the first endoprosthesis had been deployed adjacent to or even occluding the left subclavian artery no stent herniation would have occurred, as it was manifested by deploying the second endoprosthesis more proximal, close to the origin of the subclavian artery. Combination of endovascular stent and major lung resection including an aortic patch wall is not a routine procedure. We describe this intraoperative complication to share our first experience to facilitate success in some similar procedures to any other surgeons dealing with such kind of pathology.

\section{References}

1. Yildizeli B, Dartevelle PG, Fadel E, Mussot S, Chapelier A (2008) Results of primary surgery with T4 non-small cell lung cancer during a 25 -year period in a single center: the benefit is worth the risk. Ann Thorac Surg 86: 1065-1075. [Crossref]

2. Riesenman PJ, Mark AF, Mendes RR, Marston WA, Fulton JJ, et al. (2006) Coverage of the left subclavian artery during thoracic endovascular aortic repair. J Vasc Surg 45: 90-95. [Crossref]

3. Berna P, Bagan P, De Dominicis F, Dayen C, Douadi Y, et al. (2011) Aortic endostent followd by extended pneumonectomy for T4 lung cancer. Ann Thorac Surg 91: 591593. [Crossref]

Copyright: (C2017 Martínez AFH. This is an open-access article distributed under the terms of the Creative Commons Attribution License, which permits unrestricted use, distribution, and reproduction in any medium, provided the original author and source are credited. 